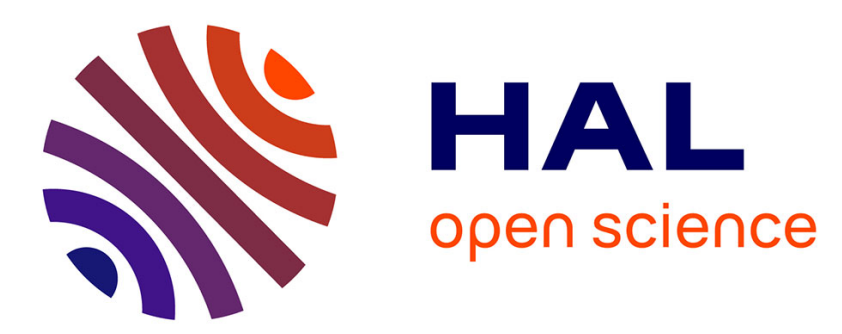

\title{
Semiclassical perturbation Stark shifts of singly charged argon spectral lines
}

\author{
Rafik Hamdi, Nabil Ben Nessib, Sylvie Sahal-Bréchot, Milan Dimitrijević
}

\section{To cite this version:}

Rafik Hamdi, Nabil Ben Nessib, Sylvie Sahal-Bréchot, Milan Dimitrijević. Semiclassical perturbation Stark shifts of singly charged argon spectral lines. Monthly Notices of the Royal Astronomical Society, 2019, 488 (2), pp.2473-2479. 10.1093/mnras/stz1835 . hal-02301744

\section{HAL Id: hal-02301744 \\ https://hal.sorbonne-universite.fr/hal-02301744}

Submitted on 30 Sep 2019

HAL is a multi-disciplinary open access archive for the deposit and dissemination of scientific research documents, whether they are published or not. The documents may come from teaching and research institutions in France or abroad, or from public or private research centers.
L'archive ouverte pluridisciplinaire HAL, est destinée au dépôt et à la diffusion de documents scientifiques de niveau recherche, publiés ou non, émanant des établissements d'enseignement et de recherche français ou étrangers, des laboratoires publics ou privés. 


\title{
Semiclassical perturbation Stark shifts of singly charged argon spectral lines
}

\author{
Rafik Hamdi, ${ }^{1,2 \star}$ Nabil Ben Nessib, ${ }^{3 \star}$ Sylvie Sahal-Bréchot ${ }^{4 \star}$ \\ and Milan S. Dimitrijevic ${ }^{4,5,6}$ \\ ${ }^{1}$ Deanship of Joint First Year, Department of Physics, Umm Al-Qura University, Makkah, Saudi Arabia \\ ${ }^{2}$ Groupe de Recherche en Physique Atomique et Astrophysique, Faculté des Sciences de Bizerte, Université de Carthage, Tunisia \\ ${ }^{3}$ Department of Physics and Astronomy, College of Science, King Saud University. PO Box 2455, Riyadh 11451, Saudi Arabia \\ ${ }^{4}$ Sorbonne Université, Observatoire de Paris, Université PSL, CNRS, LERMA, F-92190, Meudon, France \\ ${ }^{5}$ Astronomical Observatory, Volgina 7, 11060 Belgrade, Serbia \\ ${ }^{6}$ Institute Isaac Newton of Chile, Yugoslavia Branch, 11060 Belgrade, Serbia
}

Accepted 2019 June 28. Received 2019 June 7; in original form 2018 November 12

\begin{abstract}
Using semiclassical perturbation approach in impact approximation, Stark shifts for singly charged argon (Ar II) spectral lines have been calculated. Energy levels and oscillator strengths needed for this calculation were determined using Hartree-Fock method with relativistic correction (HFR). We compare our results with experimental values for 100 spectral lines. We also present results as a function of temperature for 161 spectral lines for collisions with electrons, protons, singly charged helium and singly charged argon. This work extends our previous one.
\end{abstract}

Key words: atomic data - atomic processes - line: formation.

\section{INTRODUCTION}

Stark broadening parameters (width and shift) are of interest for diagnostic and investigation of laboratory and stellar plasmas. Stark broadening parameters are needed for a number of problems in astrophysics like, e.g. for the determination of abundance in stellar atmospheres, radiative transfer calculations, stellar spectra analysis, and synthesis, etc. Dimitrijević \& Sahal-Bréchot (2014), reviews the applications of Stark broadening data determined using a semiclassical perturbations approach. This review shows that the main users of semiclassical perturbation Stark broadening data are stellar physicists studying A-type stars, B-type stars, and white dwarfs. The importance of Stark broadening mechanism for different kind of stellar atmospheres is shown in various papers (Simić et al. 2005; Hamdi et al. 2008, 2014; Dimitrijević et al. 2015; Simić, Dimitrijević \& Sahal-Bréchot 2013; Majlinger, Simić \& Dimitrijević 2017). Vennes, Kawka \& Németh (2011), studied the influence of Stark shift on the abundance in the atmospheres of the heavily polluted DAZ white dwarf GALEX J1931+0117. Using model atmosphere method, Khokhlova (1994) illustrates the significance of Stark shifts of spectral lines by collisions with electrons for element abundance determination in the atmospheres of magnetic, chemically peculiar stars. Stark shifts are also needed

^E-mail: Rafik.Hamdi@istls.rnu.tn (RH); nbennessib@ksu.edu.sa (NBN); to make accurate determination of the gravitational redshifts in white dwarfs spectra (Wiese \& Kelleher 1971).

With the development of space astronomy and observational techniques, argon is more and more interesting for astrophysics since now it is observed in various ionization stages in different astrophysical objects. For example, Ar VII is observed by Werner, Rauch \& Kruk (2018) in the hot white dwarf in the peculiar binary nucleus of the planetary nebula EGB 6. Ar VII $\lambda=1065.55 \AA$ spectral line is observed in ultraviolet spectra of hot PG 1159 and extremely hot helium rich white dwarfs (Werner, Rauch \& Kruk 2016, 2017). Ar III and Ar II spectral lines are used in the determination of argon abundance in B-type star and subdwarf atmospheres (Heber \& Edelmann 2004; Blanchette et al. 2008; Lanz et al. 2008; Fossati et al. 2009).

In our recent works, Hamdi et al. (2017) and Hamdi et al. (2018), we have reported Stark widths for 300 spectral lines of singly charged argon (Ar II). Our Stark widths were compared with experimental results and good agreements were found for most spectral lines. In order to complete the set of Stark broadening parameters of ArII spectral lines, we report here Stark shifts. Stark shifts are calculated using semiclassical perturbation approach (SCP) (Sahal-Bréchot 1969a,b) and a set of atomic data is calculated using Hartree-Fock method with relativistic corrections (Cowan 1981). Our results are compared with experimental results for 100 spectral lines. Shifts by collisions with ionic perturbers are also taken into account. We also present results as a function of temperature for 161 spectral lines for collisions with electrons, protons, singly charged helium, and singly charged argon. In 
previous works (Hamdi et al. 2011, 2013, 2014), the same method was used and we have determined Stark broadening parameters for 114 spectral lines of $\mathrm{Pb}$ IV and 32 spectral lines of Ar III.

\section{THE IMPACT SEMICLASSICAL PERTURBATION METHOD}

A detailed description of this formalism with all the innovations is given in Sahal-Bréchot (1969a, b, 1974, 1991), Fleurier, SahalBréchot \& Chapelle (1977), Dimitrijević, Sahal-Bréchot \& Bommier (1991), Dimitrijević \& Sahal-Bréchot (1996), Sahal-Bréchot, Dimitrijević \& Ben Nessib (2014), and earlier papers. The profile $F(\omega)$ is Lorentzian for isolated lines:

$F(\omega)=\frac{w / \pi}{\left(\omega-\omega_{i f}-d\right)^{2}+w^{2}}$,

where

$\omega_{i f}=\frac{E_{i}-E_{f}}{\hbar}$

$i$ and $f$ denote the initial and final states and $E_{i}$ and $E_{f}$ their corresponding energies and $w$ is the half width.

The shift $(d)$ (in angular frequency units) of an electron-impact shifted spectral line can be expressed as:

$d=N \int v f(v) \mathrm{d} v \int_{R_{3}}^{R_{\mathrm{D}}} 2 \pi \rho \mathrm{d} \rho \sin \left(2 \varphi_{\mathrm{p}}\right)$,

where $N$ is the electron density, $f(v)$ the Maxwellian velocity distribution function for electrons, $\rho$ denotes the impact parameter of the incoming electron.

The phase shift $\varphi_{\mathrm{p}}$ due to the polarization potential $\left(r^{-4}\right)$, is given in section 3 of chapter 2 in Sahal-Bréchot (1969a) and $R_{\mathrm{D}}$ is the Debye radius. The cut-off $R_{3}$ is described in section 1 of chapter 3 in Sahal-Bréchot (1969b).

Energy levels and oscillator strengths needed for SCP calculations of Stark shifts are obtained using Hartree-Fock method with relativistic corrections (HFR) (Cowan 1981). We use as for the width calculations an atomic model including 24 configurations: $3 \mathrm{~s}^{2} 3 \mathrm{p}^{5} ; 3 \mathrm{~s}^{2} 3 \mathrm{p}^{4} n l(n l=4 \mathrm{p}, 4 \mathrm{f}, 5 \mathrm{p}, 5 \mathrm{f}, 6 \mathrm{p}, 6 \mathrm{f}, 6 \mathrm{~h}, 7 \mathrm{p}, 7 \mathrm{f}, 7 \mathrm{~h}$ ) (odd

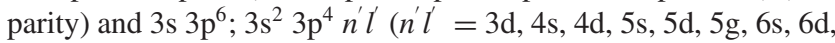
$6 \mathrm{~g}, 7 \mathrm{~s}, 7 \mathrm{~d}, 7 \mathrm{~g}$ ) (even parity). We use an ab initio procedure.

In order to introduce a correction to the shifts due to the difference between calculated and experimental wavelengths, we have used equation (8) of Hamdi et al. (2013).

\section{STARK SHIFTS}

In Table 1, we present our Stark shifts along with experimental results of Djeniže et al. (1989), Dzierzega \& Musiol (1994), Aparicio et al. (1998), Djurović et al. (2013), and the theoretical results of Griem (1974).

In the first five columns of Table 1, we give the transition array, transition, wavelength, electron temperature $(T)$, and electron density $\left(N_{\mathrm{e}}\right)$. In column 6 of Table 1 , we show the experimental Stark shifts $\left(d_{\mathrm{m}}\right)$. In the columns 7 and column 8, we present our electron-impact Stark shifts $\left(d_{\mathrm{e}}\right)$ and ion impact Stark shifts $\left(d_{\mathrm{i}}\right)$ calculated using semiclassical perturbation approach as described in Section 2. Ionic perturbers are $\mathrm{Ar}^{+}$ions. We have checked that the impact approximation is valid for collisions with ions in the conditions of temperatures and densities of these experiments. In the case when the impact approximation is not valid, the value of the shift is not given. As for the transition $\left({ }^{3} \mathrm{P}\right) 4 \mathrm{p}^{4} \mathrm{D}_{7 / 2}^{\mathrm{o}}-\left({ }^{3} \mathrm{P}\right) 4 \mathrm{~d}$ ${ }^{4} \mathrm{~F}_{9 / 2}(\lambda=3588.44 \AA)$, for which ions impact Stark shifts are not given when we compare with Djeniže et al. (1989) and Dzierzega \& Musiol (1994). The ratios $\left(d_{\mathrm{m}} / d\right)$ between the experimental and our calculated Stark shifts are shown in column 9, where $d=d_{\mathrm{e}}+d_{\mathrm{i}}$ is the total shift. In column 10, we present the Stark shifts of Griem (1974). The accuracies of the experimental results are presented in column 11. We use code letters from critical reviews of Konjević et al. (2002) and Lesage (2009) which indicate the following:

$$
\begin{aligned}
& \mathrm{A}=\text { uncertainties within } 15 \text { percent } \\
& \mathrm{B}+=\text { uncertainties within } 23 \text { percent } \\
& \mathrm{B}=\text { uncertainties within } 30 \text { percent } \\
& \mathrm{C}+=\text { uncertainties within } 40 \text { percent } \\
& \mathrm{C}=\text { uncertainties within } 50 \text { percent } \\
& \mathrm{D}=\text { uncertainties larger than } 50 \text { per cent. }
\end{aligned}
$$

Finally, in the last column of Table 1, a label for each set of experimental results is given. In this work, we are interested to transitions of the type: $4 p-4 d$ and $4 p-5 s$.

In our calculations, only electric dipole transitions (E1) are taken into account. We have checked that the use of magnetic dipole (M1) and electric quadrupole (E2) transitions have no influence on the results. In Table 2, we compare our calculated wavelengths and oscillators strengths and the values taken from NIST data base for some transitions used in our calculation. We can see from Table 2 that our calculated wavelengths agree with NIST ones within 2.4 per cent in average and the agreement between our oscillators strengths and NIST ones is 22 per cent in average.

The agreement of our Stark shifts with the experimental results is generally good for $\left({ }^{3} \mathrm{P}\right) 4 \mathrm{p}-\left({ }^{3} \mathrm{P}\right) 4 \mathrm{~d}$ transition array. Comparing with Djurović et al. (2013), the agreement is good (27 percent in average) except for four transitions belonging to ${ }^{2} \mathrm{D}-{ }^{2} \mathrm{P},{ }^{2} \mathrm{P}-$ ${ }^{2} \mathrm{P}$, and ${ }^{2} \mathrm{~S}-{ }^{2} \mathrm{D}$ multiplet for which our Stark shifts are small comparing to the experimental results of Djurović et al. (2013). Our results also agree well with Aparicio et al. (1998) (27 percent in average) except for three transitions. For the transition : ${ }^{4} \mathrm{D}_{1 / 2}^{\mathrm{o}}$ ${ }^{4} \mathrm{D}_{1 / 2}(\lambda=3880.33 \AA)$, the ratio $\frac{d_{\mathrm{m}}}{d}$ varies from 0.39 to 0.42 when we compare with Aparicio et al. (1998), on the other hand the ratio $\frac{d_{\mathrm{m}}}{d}$ is equal 1.06 when we compare with Djurović et al. (2013). For the transition ${ }^{4} \mathrm{D}_{5 / 2}^{\mathrm{o}}-{ }^{4} \mathrm{~F}_{7 / 2}(\lambda=3576.62 \AA)$, our result disagree with Aparicio et al. (1998) but the agreement is good with Djeniže et al. (1989). For $\left({ }^{1} \mathrm{D}\right) 4 \mathrm{p}-\left({ }^{1} \mathrm{D}\right) 4 \mathrm{~d}$ transition array, the better agreement was found with Djeniže et al. (1989) (35 per cent in average). The agreement with Djurović et al. (2013) and Aparicio et al. (1998) is worse, in fact our results are generally two and three times larger than the experimental values. Stark shifts due to collision with ions are not given for all $\left({ }^{1} \mathrm{D}\right) 4 \mathrm{p}-\left({ }^{1} \mathrm{D}\right) 4 \mathrm{~d}$ transitions since impact approximation is not valid. For $\left({ }^{3} \mathrm{P}\right) 4 \mathrm{p}-\left({ }^{3} \mathrm{P}\right) 5 \mathrm{~s}$ transition array, our Stark shifts agree well with the experimental results. In fact, the agreement with Aparicio et al. (1998) is within 31 percent in average. The difference with Djurović et al. (2013) do not exceed 32 percent.

\section{LARGE-SCALE CALCULATIONS}

We present our large-scale calculations as a function of temperatures in Table 3. The complete obtained results for the 161 spectral lines of Ar II are given as additional data in electronic form in the online journal. Here, only a sample of the results is provided in order to demonstrate the content of additional data and their form. The calculations have been made for a perturber density of 
Table 1. Our electron-impact Stark shifts $\left(d_{\mathrm{e}}\right)$ and ion impact Stark shifts $\left(d_{\mathrm{i}}\right)$ calculated using SCP approach in impact approximation (Sahal-Bréchot 1969a,b) compared with experimental Stark shifts $\left(d_{\mathrm{m}}\right)$ and the Stark shifts of Griem (1974) $\left(d_{\mathrm{G}}\right) . d=d_{\mathrm{e}}+d_{\mathrm{i}}$ is the total shift. Transitions, wavelengths, electron temperature $(T)$, and electron density $\left(N_{\mathrm{e}}\right)$ are also given. Ref.: a: Djeniže et al. (1989); b: Dzierzega \& Musiol (1994); c: Aparicio et al. (1998); d: Djurović et al. (2013). All wavelengths are taken from NIST database (Kramida et al. 2018).

\begin{tabular}{|c|c|c|c|c|c|c|c|c|c|c|c|}
\hline $\begin{array}{l}\text { Transition } \\
\text { array }\end{array}$ & Transition & $\begin{array}{c}\lambda \\
(\AA)\end{array}$ & $\begin{array}{c}T \\
\left(10^{3} \mathrm{~K}\right)\end{array}$ & $\begin{array}{c}N_{\mathrm{e}} \\
\left(10^{17} \mathrm{~cm}^{-3}\right)\end{array}$ & $\begin{array}{c}d_{\mathrm{m}} \\
(\mathrm{pm})\end{array}$ & $\begin{array}{c}d_{\mathrm{e}} \\
(\mathrm{pm})\end{array}$ & $\begin{array}{c}d_{\mathrm{i}} \\
(\mathrm{pm})\end{array}$ & $d_{\mathrm{m}} / d$ & $\begin{array}{l}d_{\mathrm{G}} \\
(\mathrm{pm})\end{array}$ & Acc. & Ref. \\
\hline \multirow[t]{41}{*}{$\left({ }^{3} \mathrm{P}\right) 4 \mathrm{p}-\left({ }^{3} \mathrm{P}\right) 4 \mathrm{~d}$} & ${ }^{4} \mathrm{P}_{3 / 2}^{\mathrm{o}}-{ }^{4} \mathrm{D}_{5 / 2}$ & 3514.39 & 22.0 & 1.00 & 14.26 & 18.5 & 2.60 & 0.68 & & A & d \\
\hline & ${ }^{4} \mathrm{P}_{5 / 2}^{\mathrm{o}}-{ }^{4} \mathrm{D}_{5 / 2}$ & 3476.75 & 26.0 & 1.00 & 20.4 & 30.8 & 4.61 & 0.58 & & $\mathrm{~B}+$ & $\mathrm{a}$ \\
\hline & & & 22.0 & 1.00 & 17.65 & 18.3 & 2.55 & 0.85 & & A & $\mathrm{d}$ \\
\hline & ${ }^{4} \mathrm{P}_{1 / 2}^{\mathrm{o}}-{ }^{4} \mathrm{D}_{1 / 2}$ & 3509.78 & 22.0 & 1.00 & 13.40 & 20.2 & 2.71 & 0.58 & & A & $\mathrm{d}$ \\
\hline & ${ }^{4} \mathrm{P}_{3 / 2}^{\mathrm{o}}-{ }^{4} \mathrm{P}_{3 / 2}$ & 3212.52 & 22.0 & 1.00 & 16.55 & 18.3 & 2.87 & 0.78 & 24.0 & A & $\mathrm{d}$ \\
\hline & ${ }^{4} \mathrm{P}_{1 / 2}^{\mathrm{o}}-{ }^{4} \mathrm{P}_{1 / 2}$ & 3281.71 & 22.0 & 1.00 & 25.85 & 20.8 & 3.25 & 1.07 & 25.1 & A & $\mathrm{d}$ \\
\hline & ${ }^{4} \mathrm{P}_{5 / 2}^{\mathrm{o}}-{ }^{4} \mathrm{P}_{3 / 2}$ & 3181.04 & 22.0 & 1.00 & 18.78 & 18.0 & 2.82 & 0.90 & 23.8 & A & $\mathrm{d}$ \\
\hline & ${ }^{4} \mathrm{P}_{3 / 2}^{\mathrm{o}}-{ }^{4} \mathrm{P}_{1 / 2}$ & 3243.69 & 22.0 & 1.00 & 18.37 & 20.3 & 3.17 & 0.78 & 24.5 & A & $\mathrm{d}$ \\
\hline & ${ }^{4} \mathrm{P}_{3 / 2}^{\mathrm{o}}-{ }^{4} \mathrm{P}_{5 / 2}$ & 3169.67 & 26.0 & 1.76 & 21.5 & 21.9 & & & 42.2 & $\mathrm{~B}+$ & $\mathrm{a}$ \\
\hline & & & 22.0 & 1.00 & 16.80 & 17.2 & 2.78 & 0.84 & 23.3 & $\mathrm{~B}$ & $\mathrm{~d}$ \\
\hline & ${ }^{4} \mathrm{D}_{7 / 2}^{\mathrm{o}}-{ }^{4} \mathrm{D}_{7 / 2}$ & 3780.84 & 26.0 & 1.76 & 15.4 & 35.2 & 5.52 & 0.38 & 49.7 & B & $\mathrm{a}$ \\
\hline & & & $18.4-26.5$ & 1.00 & 18.8 & $22.2-20.0$ & $2.85-3.24$ & $0.75-0.81$ & $29.8-29.8$ & B & $\mathrm{c}$ \\
\hline & & & 22.0 & 1.00 & 17.80 & 21.5 & 3.04 & 0.73 & 28.3 & A & $\mathrm{d}$ \\
\hline & ${ }^{4} \mathrm{D}_{5 / 2}^{\mathrm{o}}-{ }^{4} \mathrm{D}_{5 / 2}$ & 3826.81 & $18.4-26.5$ & 1.00 & 18.8 & $23.0-21.0$ & $2.95-3.29$ & $0.72-0.77$ & $30.3-29.4$ & $\mathrm{~B}+$ & $\mathrm{c}$ \\
\hline & & & 22.0 & 1.00 & 20.13 & 21.9 & 3.10 & 0.80 & 28.3 & A & $\mathrm{d}$ \\
\hline & ${ }^{4} \mathrm{D}_{3 / 2}^{\mathrm{o}}-{ }^{4} \mathrm{D}_{3 / 2}$ & 3872.14 & $18.4-26.5$ & 1.00 & 20.0 & $24.5-22.2$ & $3.06-3.43$ & $0.73-0.78$ & $31.3-29.9$ & $\mathrm{~B}+$ & $\mathrm{c}$ \\
\hline & ${ }^{4} \mathrm{D}_{1 / 2}^{\mathrm{o}}-{ }^{4} \mathrm{D}_{1 / 2}$ & 3880.33 & $18.4-26.5$ & 1.00 & 11.0 & $25.3-22.7$ & $3.13-3.51$ & $0.39-0.42$ & $31.4-29.7$ & $\mathrm{~B}+$ & $\mathrm{c}$ \\
\hline & & & 22.0 & 1.00 & 28.95 & 24.0 & 3.30 & 1.06 & 29.5 & B & $\mathrm{d}$ \\
\hline & ${ }^{4} \mathrm{D}_{7 / 2}^{\mathrm{o}}-{ }^{4} \mathrm{D}_{5 / 2}$ & 3763.50 & $18.4-26.5$ & 1.00 & 16.4 & $21.6-19.6$ & $2.82-3.14$ & $0.67-0.72$ & $30.9-29.3$ & $\mathrm{~B}+$ & $\mathrm{c}$ \\
\hline & & & 22.0 & 1.00 & 16.40 & 20.7 & 2.97 & 0.69 & 27.8 & B & $\mathrm{d}$ \\
\hline & ${ }^{4} \mathrm{D}_{5 / 2}^{\mathrm{o}}-{ }^{4} \mathrm{D}_{3 / 2}$ & 3799.38 & $18.4-26.5$ & 1.00 & 20.7 & $24.2-21.8$ & $2.98-3.36$ & $0.76-0.82$ & $30.0-28.7$ & $\mathrm{~B}+$ & $\mathrm{c}$ \\
\hline & & & 22.0 & 1.00 & 21.51 & 22.9 & 3.14 & 0.83 & 28.3 & A & $\mathrm{d}$ \\
\hline & & & 11.52 & 1.79 & 41.6 & 52.7 & - & 0.79 & & $\mathrm{~B}+$ & $\mathrm{b}$ \\
\hline & & & 13.03 & 1.10 & 22.7 & 32.7 & - & 0.69 & & $\mathrm{~B}+$ & $\mathrm{b}$ \\
\hline & & & 13.88 & 1.39 & 29.4 & 41.1 & - & 0.71 & & $\mathrm{~B}+$ & $\mathrm{b}$ \\
\hline & ${ }^{4} D_{5 / 2}^{o}-{ }^{4} F_{7 / 2}$ & 3576.62 & 26.0 & 1.76 & 33.3 & 38.6 & - & 0.86 & & $\mathrm{C}$ & $\mathrm{a}$ \\
\hline & & & $18.4-26.5$ & 1.00 & 56.7 & $24.5-21.9$ & $3.23-3.58$ & $2.04-2.23$ & & $\mathrm{C}$ & $\mathrm{c}$ \\
\hline & ${ }^{4} \mathrm{D}_{3 / 2}^{\mathrm{o}}-{ }^{4} \mathrm{~F}_{5 / 2}$ & 3582.35 & $18.4-26.5$ & 1.00 & 50.7 & $23.5-21.1$ & $3.14-3.48$ & $1.90-2.06$ & & $\mathrm{~B}+$ & $\mathrm{c}$ \\
\hline & ${ }^{4} \mathrm{D}_{5 / 2}^{\mathrm{o}}-{ }^{4} \mathrm{~F}_{5 / 2}$ & 3519.99 & 22.0 & 1.00 & 19.59 & 21.7 & 3.19 & 0.79 & & A & $\mathrm{d}$ \\
\hline & ${ }^{4} \mathrm{D}_{3 / 2}^{\mathrm{o}}-{ }^{4} \mathrm{~F}_{3 / 2}$ & 3548.51 & 22.0 & 1.00 & 19.28 & 21.5 & 3.19 & 0.78 & & B & $\mathrm{d}$ \\
\hline & ${ }^{4} \mathrm{D}_{1 / 2}^{\mathrm{o}}-{ }^{4} \mathrm{P}_{3 / 2}$ & 3565.03 & 26.0 & 1.76 & 30.8 & 36.7 & - & 0.84 & & $\mathrm{~B}+$ & $\mathrm{a}$ \\
\hline & ${ }^{4} \mathrm{D}_{7 / 2}^{\mathrm{o}}-{ }^{4} \mathrm{P}_{5 / 2}$ & 3370.91 & 22.0 & 1.00 & 18.99 & 18.9 & 3.14 & 0.86 & & A & $\mathrm{d}$ \\
\hline & ${ }^{4} \mathrm{D}_{5 / 2}^{\mathrm{o}}-{ }^{4} \mathrm{P}_{5 / 2}$ & 3421.61 & 22.0 & 1.00 & 21.30 & 19.9 & 3.25 & 0.92 & & A & $\mathrm{d}$ \\
\hline & ${ }^{4} \mathrm{D}_{3 / 2}^{\mathrm{o}}-{ }^{2} \mathrm{~F}_{5 / 2}$ & 3397.90 & 22.0 & 1.00 & 21.21 & 22.8 & 4.00 & 0.79 & & A & $\mathrm{d}$ \\
\hline & ${ }^{4} \mathrm{D}_{5 / 2}^{\mathrm{o}}-{ }^{2} \mathrm{~F}_{7 / 2}$ & 3430.42 & 22.0 & 1.00 & 21.14 & 24.7 & 4.12 & 0.73 & & A & $\mathrm{d}$ \\
\hline & ${ }^{4} \mathrm{D}_{5 / 2}^{\mathrm{o}}-{ }^{2} \mathrm{D}_{5 / 2}$ & 2865.84 & 22.0 & 1.00 & $\left|d_{\mathrm{m}}\right|<2.5$ & -5.51 & - & & & & $\mathrm{d}$ \\
\hline & ${ }^{2} \mathrm{D}_{5 / 2}^{\mathrm{o}}-{ }^{4} \mathrm{D}_{3 / 2}$ & 3958.38 & 22.0 & 1.00 & 17.52 & 25.5 & 3.47 & 0.60 & & A & $\mathrm{d}$ \\
\hline & ${ }^{2} \mathrm{D}_{5 / 2}^{\mathrm{o}}-{ }^{4} \mathrm{D}_{5 / 2}$ & 3988.16 & $18.4-26.5$ & 1.00 & 34.1 & $25.6-23.3$ & $3.27-3.66$ & $1.18-1.26$ & & $\mathrm{~B}+$ & $\mathrm{c}$ \\
\hline & & & 22.0 & 1.00 & 22.25 & 24.5 & 3.42 & 0.80 & & B & $\mathrm{d}$ \\
\hline & ${ }^{2} \mathrm{D}_{3 / 2}^{\mathrm{o}}-{ }^{4} \mathrm{D}_{1 / 2}$ & 4031.38 & 22.0 & 1.00 & 21.28 & 28.16 & 3.73 & 0.67 & & B & $\mathrm{d}$ \\
\hline & ${ }^{2} \mathrm{D}_{5 / 2}^{\mathrm{o}}-{ }^{2} \mathrm{D}_{5 / 2}$ & 2955.39 & 22.0 & 1.00 & 3.02 & -5.62 & - & 0.54 & & $\mathrm{C}$ & $\mathrm{d}$ \\
\hline
\end{tabular}


Table 1 - continued

\begin{tabular}{|c|c|c|c|c|c|c|c|c|c|c|c|}
\hline $\begin{array}{l}\text { Transition } \\
\text { array }\end{array}$ & Transition & $\begin{array}{c}\lambda \\
(\AA)\end{array}$ & $\begin{array}{c}T \\
\left(10^{3} \mathrm{~K}\right)\end{array}$ & $\begin{array}{c}N_{\mathrm{e}} \\
\left(10^{17} \mathrm{~cm}^{-3}\right)\end{array}$ & $\begin{array}{c}d_{\mathrm{m}} \\
(\mathrm{pm})\end{array}$ & $\begin{array}{c}d_{\mathrm{e}} \\
(\mathrm{pm})\end{array}$ & $\begin{array}{c}d_{\mathrm{i}} \\
(\mathrm{pm})\end{array}$ & $d_{\mathrm{m}} / d$ & $\begin{array}{c}d_{\mathrm{G}} \\
(\mathrm{pm})\end{array}$ & Acc. & Ref. \\
\hline & ${ }^{2} \mathrm{D}_{5 / 2}^{\mathrm{o}}-{ }^{2} \mathrm{~F}_{5 / 2}$ & 3464.13 & 26.0 & 1.76 & 36.0 & 40.7 & - & 0.88 & 56.3 & $\mathrm{~B}+$ & $\mathrm{a}$ \\
\hline & & & 22.0 & 1.00 & 26.04 & 24.5 & 4.17 & 0.91 & 31.4 & A & d \\
\hline & ${ }^{2} \mathrm{D}_{3 / 2}^{\mathrm{o}}-{ }^{2} \mathrm{~F}_{5 / 2}$ & 3545.60 & 22.0 & 1.00 & 25.08 & 26.6 & 2.71 & 0.91 & 33.0 & B & d \\
\hline & ${ }^{2} \mathrm{D}_{3 / 2}^{\mathrm{o}}-{ }^{2} \mathrm{P}_{1 / 2}$ & 3273.32 & 22.0 & 1.00 & 28.59 & 6.21 & - & 4.60 & & A & d \\
\hline & ${ }^{2} \mathrm{D}_{3 / 2}^{\mathrm{o}}-{ }^{2} \mathrm{P}_{3 / 2}$ & 3204.32 & 22.0 & 1.00 & 17.94 & 2.55 & - & 7.04 & & $\mathrm{~A}$ & d \\
\hline & ${ }^{2} \mathrm{P}_{3 / 2}^{\mathrm{o}}-{ }^{2} \mathrm{~F}_{5 / 2}$ & 3655.28 & $18.4-26.5$ & 1.00 & 17.4 & $27.8-24.9$ & $4.26-4.69$ & $0.54-0.59$ & & $\mathrm{~B}+$ & $\mathrm{c}$ \\
\hline & & & 22.0 & 1.00 & 22.06 & 26.5 & 4.54 & 0.71 & & B & d \\
\hline & ${ }^{2} \mathrm{P}_{1 / 2}^{\mathrm{o}}-{ }^{2} \mathrm{P}_{1 / 2}$ & 3307.23 & 26.0 & 1.76 & 30.8 & 11.4 & - & 2.70 & 88.0 & $\mathrm{~B}+$ & $\mathrm{a}$ \\
\hline & & & 22.0 & 1.00 & 35.02 & 6.90 & - & 5.07 & 52.3 & B & d \\
\hline & ${ }^{2} \mathrm{P}_{3 / 2}^{\mathrm{o}}-{ }^{2} \mathrm{P}_{3 / 2}$ & 3293.64 & 26.0 & 1.76 & 23.0 & 2.05 & - & 9.2 & 88.5 & $\mathrm{~B}+$ & $\mathrm{a}$ \\
\hline & ${ }^{2} \mathrm{P}_{1 / 2}^{\mathrm{o}}-{ }^{2} \mathrm{P}_{3 / 2}$ & 3236.81 & 22.0 & 1.00 & 18.88 & 0.90 & - & 21.0 & 49.7 & B & d \\
\hline & ${ }^{2} \mathrm{P}_{3 / 2}^{\mathrm{o}}-{ }^{2} \mathrm{P}_{1 / 2}$ & 3366.58 & 22.0 & 1.00 & 27.60 & 7.30 & - & 3.78 & 54.1 & B & d \\
\hline & ${ }^{2} \mathrm{P}_{3 / 2}^{\mathrm{o}}-{ }^{2} \mathrm{D}_{5 / 2}$ & 3093.40 & 22.0 & 1.00 & $\left|d_{\mathrm{m}}\right|<2.5$ & -5.76 & - & & & & d \\
\hline & ${ }^{2} \mathrm{P}_{1 / 2}^{\mathrm{o}}-{ }^{2} \mathrm{D}_{3 / 2}$ & 3028.91 & 22.0 & 1.00 & $\left|d_{\mathrm{m}}\right|<2.5$ & -1.30 & - & & & & d \\
\hline & ${ }^{4} \mathrm{~S}_{3 / 2}^{\mathrm{o}}-{ }^{4} \mathrm{~F}_{3 / 2}$ & 3952.73 & $18.4-26.5$ & 1.00 & 27.6 & $26.7-24.2$ & $3.70-4.11$ & $0.91-0.97$ & & $\mathrm{~B}+$ & $\mathrm{c}$ \\
\hline & & & 22.0 & 1.00 & 24.73 & 25.5 & 3.91 & 0.84 & & A & d \\
\hline & ${ }^{4} S_{3 / 2}^{o}-{ }^{4} P_{5 / 2}$ & 3868.53 & 26.0 & 1.76 & 35.9 & 39.6 & - & 0.91 & 60.8 & $\mathrm{~B}+$ & $\mathrm{a}$ \\
\hline & & & $18.4-26.5$ & 1.00 & 30.2 & $24.8-22.5$ & $3.86-4.27$ & $1.05-1.13$ & $35.9-34.3$ & $\mathrm{~B}+$ & $\mathrm{c}$ \\
\hline & & & 22.0 & 1.00 & 36.45 & 23.7 & 4.06 & 1.31 & 33.8 & B & $\mathrm{d}$ \\
\hline & ${ }^{4} \mathrm{~S}_{3 / 2}^{\mathrm{o}}-{ }^{4} \mathrm{P}_{3 / 2}$ & 3932.55 & $18.4-26.5$ & 1.00 & 25.8 & $26.4-24.1$ & $4.01-4.45$ & $0.85-0.90$ & $37.4-35.4$ & $\mathrm{~B}+$ & $\mathrm{c}$ \\
\hline & & & 22.0 & 1.00 & 27.34 & 25.4 & 4.25 & 0.92 & 35.1 & A & d \\
\hline & ${ }^{4} \mathrm{~S}_{3 / 2}^{\mathrm{o}}-{ }^{4} \mathrm{P}_{1 / 2}$ & 3979.36 & $18.4-26.5$ & 1.00 & 23.1 & $30.2-27.3$ & $4.39-4.83$ & $0.67-0.72$ & $37.9-36.1$ & $\mathrm{~B}+$ & $\mathrm{c}$ \\
\hline & ${ }^{2} \mathrm{~S}_{1 / 2}^{\mathrm{o}}-{ }^{2} \mathrm{P}_{3 / 2}$ & 3388.53 & 26.0 & 1.76 & 25.5 & -0.964 & - & -26.5 & & $\mathrm{~B}+$ & $\mathrm{a}$ \\
\hline & ${ }^{2} \mathrm{~S}_{1 / 2}^{\mathrm{o}}-{ }^{2} \mathrm{D}_{3 / 2}$ & 3161.37 & 22.0 & 1.00 & 5.58 & -2.53 & - & -2.21 & & $\mathrm{C}$ & d \\
\hline \multirow[t]{2}{*}{$\left({ }^{1} \mathrm{D}\right) 4 \mathrm{p}-\left({ }^{3} \mathrm{P}\right) 4 \mathrm{~d}$} & ${ }^{2} \mathrm{P}_{3 / 2}^{\mathrm{o}}-{ }^{2} \mathrm{D}_{5 / 2}$ & 4914.31 & $18.4-26.5$ & 1.00 & -70.7 & $(-23.3)-(-19.8)$ & - & $3.03-3.57$ & & B & $\mathrm{c}$ \\
\hline & ${ }^{2} \mathrm{D}_{5 / 2}^{\mathrm{o}}-{ }^{2} \mathrm{P}_{3 / 2}$ & 5812.76 & $18.4-26.5$ & 1.00 & 79.9 & $7.62-5.76$ & - & $10.5-13.9$ & & $\mathrm{~B}+$ & $\mathrm{c}$ \\
\hline \multirow[t]{25}{*}{$\left({ }^{1} \mathrm{D}\right) 4 \mathrm{p}-\left({ }^{1} \mathrm{D}\right) 4 \mathrm{~d}$} & ${ }^{2} \mathrm{~F}_{7 / 2}^{\mathrm{o}}-{ }^{2} \mathrm{G}_{9 / 2}$ & 3561.03 & 22.0 & 1.00 & 15.00 & 23.2 & - & 0.65 & & B & d \\
\hline & ${ }^{2} \mathrm{~F}_{7 / 2}^{\mathrm{o}}-{ }^{2} \mathrm{~F}_{7 / 2}$ & 3376.44 & 26.0 & 1.76 & 69.0 & 56.6 & - & 1.22 & & $\mathrm{~B}+$ & $\mathrm{a}$ \\
\hline & & & 22.0 & 1.00 & 30.88 & 34.3 & - & 0.90 & & $\mathrm{~A}$ & $\mathrm{~d}$ \\
\hline & ${ }^{2} \mathrm{~F}_{5 / 2}^{\mathrm{o}}-{ }^{2} \mathrm{~F}_{5 / 2}$ & 3350.92 & 22.0 & 1.00 & 22.65 & 35.6 & - & 0.64 & & A & d \\
\hline & ${ }^{2} \mathrm{~F}_{7 / 2}^{\mathrm{o}}-{ }^{2} \mathrm{~F}_{5 / 2}$ & 3365.52 & 22.0 & 1.00 & 21.87 & 35.5 & - & 0.62 & & A & d \\
\hline & ${ }^{2} \mathrm{~F}_{5 / 2}^{\mathrm{o}}-{ }^{2} \mathrm{D}_{5 / 2}$ & 3414.46 & 22.0 & 1.00 & 27.28 & 44.2 & - & 0.62 & & B & d \\
\hline & ${ }^{2} \mathrm{P}_{3 / 2}^{\mathrm{o}}-{ }^{2} \mathrm{P}_{3 / 2}$ & 3660.44 & $18.4-26.5$ & 1.00 & 15.4 & $79.8-74.3$ & - & $0.19-0.21$ & & $\mathrm{~B}+$ & $\mathrm{c}$ \\
\hline & & & 22.0 & 1.00 & 21.43 & 75.9 & - & 0.28 & & B & d \\
\hline & ${ }^{2} \mathrm{P}_{3 / 2}^{\mathrm{o}}-{ }^{2} \mathrm{P}_{1 / 2}$ & 3671.00 & 22.0 & 1.00 & 25.25 & 73.7 & - & 0.34 & & $\mathrm{C}$ & d \\
\hline & ${ }^{2} \mathrm{P}_{1 / 2}^{\mathrm{o}}-{ }^{2} \mathrm{P}_{1 / 2}$ & 3754.05 & 22.0 & 1.00 & 30.24 & 75.9 & - & 0.40 & & A & d \\
\hline & ${ }^{2} \mathrm{P}_{3 / 2}^{\mathrm{o}}-{ }^{2} \mathrm{D}_{5 / 2}$ & 3639.83 & 22.0 & 1.00 & 26.20 & 45.5 & - & 0.58 & & A & $\mathrm{d}$ \\
\hline & ${ }^{2} \mathrm{P}_{1 / 2}^{\mathrm{o}}-{ }^{2} \mathrm{~S}_{1 / 2}$ & 3082.98 & 22.0 & 1.00 & -12.61 & 27.0 & - & -0.47 & & B & $\mathrm{d}$ \\
\hline & ${ }^{2} \mathrm{D}_{5 / 2}^{\mathrm{o}}-{ }^{2} \mathrm{P}_{3 / 2}$ & 3825.67 & 22.0 & 1.00 & 26.75 & 89.0 & - & 0.30 & & $\mathrm{C}$ & d \\
\hline & ${ }^{2} \mathrm{D}_{3 / 2}^{\mathrm{o}}-{ }^{2} \mathrm{P}_{3 / 2}$ & 3819.02 & 22.0 & 1.00 & 35.83 & 86.2 & - & 0.42 & & B & $\mathrm{d}$ \\
\hline & ${ }^{2} \mathrm{D}_{5 / 2}^{\mathrm{o}}-{ }^{2} \mathrm{D}_{5 / 2}$ & 3803.17 & 26.0 & 1.76 & 53.8 & 91.6 & - & 0.59 & & B & $\mathrm{a}$ \\
\hline & & & $18.4-26.5$ & 1.00 & 37.5 & $58.9-52.3$ & - & $0.64-072$ & & B & $\mathrm{c}$ \\
\hline & & & 22.0 & 1.00 & 37.43 & 55.5 & - & 0.67 & & A & $\mathrm{d}$ \\
\hline & ${ }^{2} \mathrm{D}_{3 / 2}^{\mathrm{o}}-{ }^{2} \mathrm{D}_{5 / 2}$ & 3796.59 & $18.4-26.5$ & 1.00 & 24.1 & $57.1-50.8$ & - & $0.42-0.47$ & & $\mathrm{~B}+$ & $\mathrm{c}$ \\
\hline & & & 22.0 & 1.00 & 30.47 & 54.0 & - & 0.56 & & B & $\mathrm{d}$ \\
\hline & ${ }^{2} \mathrm{D}_{5 / 2}^{\mathrm{o}}-{ }^{2} \mathrm{~F}_{7 / 2}$ & 3737.89 & 26.0 & 1.76 & 57.7 & 71.1 & - & 0.81 & & B & $\mathrm{a}$ \\
\hline & & & $18.4-26.5$ & 1.00 & 22.2 & $45.6-40.6$ & - & $0.49-0.55$ & & $\mathrm{~B}+$ & $\mathrm{c}$ \\
\hline & & & 22.0 & 1.00 & 31.03 & 43.0 & - & 0.72 & & $\mathrm{~B}+$ & $\mathrm{d}$ \\
\hline & ${ }^{2} \mathrm{D}_{3 / 2}^{\mathrm{o}}-{ }^{2} \mathrm{~F}_{5 / 2}$ & 3718.21 & $18.4-26.5$ & 1.00 & 23.1 & $45.5-40.5$ & - & $0.51-0.57$ & & $\mathrm{~B}+$ & $\mathrm{c}$ \\
\hline & & & 22.0 & 1.00 & 23.31 & 42.9 & - & 0.54 & & B & $\mathrm{d}$ \\
\hline & ${ }^{2} \mathrm{D}_{5 / 2}^{\mathrm{o}}-{ }^{2} \mathrm{~F}_{5 / 2}$ & 3724.52 & $18.4-26.5$ & 1.00 & 14.7 & $47.1-42.0$ & - & $0.31-0.35$ & & B & $\mathrm{c}$ \\
\hline \multirow[t]{8}{*}{$\left({ }^{3} \mathrm{P}\right) 4 \mathrm{p}-\left({ }^{3} \mathrm{P}\right) 5 \mathrm{~s}$} & ${ }^{4} \mathrm{P}_{5 / 2}^{\mathrm{o}}-{ }^{4} \mathrm{P}_{5 / 2}$ & 3765.27 & $18.4-26.5$ & 1.00 & 18.2 & $31.4-28.5$ & $3.68-4.07$ & $0.52-0.56$ & $35.7-34.3$ & $\mathrm{~B}+$ & $\mathrm{c}$ \\
\hline & & & 22.0 & 1.00 & 25.26 & 28.1 & 3.88 & 0.79 & 33.7 & B & $\mathrm{d}$ \\
\hline & ${ }^{4} \mathrm{P}_{3 / 2}^{\mathrm{o}}-{ }^{4} \mathrm{P}_{3 / 2}$ & 3720.43 & $18.4-26.5$ & 1.00 & 24.9 & $31.1-28.4$ & $3.61-4.00$ & $0.72-0.77$ & $35.1-33.6$ & $\mathrm{~B}+$ & $\mathrm{c}$ \\
\hline & & & 22.0 & 1.00 & 25.68 & 29.9 & 3.81 & 0.76 & 32.9 & A & $\mathrm{d}$ \\
\hline & ${ }^{4} \mathrm{P}_{3 / 2}^{\mathrm{o}}-{ }^{4} \mathrm{P}_{1 / 2}$ & 3622.14 & $18.4-26.5$ & 1.00 & 29.8 & $29.2-26.5$ & $3.44-3.80$ & $0.91-0.98$ & $33.1-31.7$ & $\mathrm{~B}+$ & $\mathrm{c}$ \\
\hline & & & 22.0 & 1.00 & 35.99 & 28.0 & 3.63 & 1.14 & 31.3 & A & d \\
\hline & ${ }^{4} \mathrm{P}_{3 / 2}^{\mathrm{o}}-{ }^{4} \mathrm{P}_{5 / 2}$ & 3809.46 & $18.4-26.5$ & 1.00 & 27.9 & $32.2-29.2$ & $3.77-4.17$ & $0.78-0.84$ & $36.7-35.3$ & A & $\mathrm{c}$ \\
\hline & & & 22.0 & 1.00 & 35.10 & 31.0 & 3.97 & 1.00 & 34.8 & A & $\mathrm{d}$ \\
\hline
\end{tabular}


Table 1 - continued

\begin{tabular}{|c|c|c|c|c|c|c|c|c|c|c|c|}
\hline $\begin{array}{l}\text { Transition } \\
\text { array }\end{array}$ & Transition & $\begin{array}{c}\lambda \\
(\AA)\end{array}$ & $\begin{array}{c}T \\
\left(10^{3} \mathrm{~K}\right)\end{array}$ & $\begin{array}{c}N_{\mathrm{e}} \\
\left(10^{17} \mathrm{~cm}^{-3}\right)\end{array}$ & $\begin{array}{c}d_{\mathrm{m}} \\
(\mathrm{pm})\end{array}$ & $\begin{array}{c}d_{\mathrm{e}} \\
(\mathrm{pm})\end{array}$ & $\begin{array}{c}d_{\mathrm{i}} \\
(\mathrm{pm})\end{array}$ & $d_{\mathrm{m}} / d$ & $\begin{array}{c}d_{\mathrm{G}} \\
(\mathrm{pm})\end{array}$ & Acc. & Ref. \\
\hline & \multirow[t]{2}{*}{${ }^{4} \mathrm{P}_{1 / 2}^{\mathrm{o}}-{ }^{4} \mathrm{P}_{3 / 2}$} & \multirow[t]{2}{*}{3770.52} & 26.0 & 1.76 & 49.8 & 51.2 & 7.02 & 0.86 & 59.5 & $\mathrm{~B}+$ & c \\
\hline & & & 22.0 & 1.00 & 26.42 & 30.7 & 3.91 & 0.76 & 33.9 & A & $\mathrm{d}$ \\
\hline & ${ }^{4} \mathrm{P}_{1 / 2}^{\mathrm{o}}-{ }^{4} \mathrm{P}_{1 / 2}$ & 3669.60 & 22.0 & 1.00 & 28.99 & 28.7 & 3.73 & 0.89 & 32.2 & B & $\mathrm{d}$ \\
\hline & ${ }^{4} \mathrm{P}_{5 / 2}^{\mathrm{o}}-{ }^{4} \mathrm{P}_{3 / 2}$ & 3678.27 & 22.0 & 1.00 & 27.04 & 29.3 & 3.72 & 0.82 & 32.2 & B & $\mathrm{d}$ \\
\hline & ${ }^{4} \mathrm{D}_{3 / 2}^{\mathrm{o}}-{ }^{4} \mathrm{P}_{1 / 2}$ & 4033.81 & 22.0 & 1.00 & 33.42 & 34.4 & 4.49 & 0.86 & & A & $\mathrm{d}$ \\
\hline & ${ }^{4} \mathrm{D}_{5 / 2}^{\mathrm{o}}-{ }^{4} \mathrm{P}_{5 / 2}$ & 4179.30 & $18.4-26.5$ & 1.00 & 31.9 & $38.8-35.1$ & $4.60-5.08$ & $0.74-0.79$ & & A & $\mathrm{c}$ \\
\hline & ${ }^{4} \mathrm{D}_{5 / 2}^{\mathrm{o}}-{ }^{4} \mathrm{P}_{5 / 2}$ & 4179.30 & $18.4-26.5$ & 1.00 & 32.3 & $38.3-34.8$ & $4.49-4.97$ & $0.75-0.81$ & & A & $\mathrm{c}$ \\
\hline & \multirow{2}{*}{${ }^{4} \mathrm{D}_{3 / 2}^{\mathrm{o}}-{ }^{2} \mathrm{P}_{3 / 2}$} & \multirow[t]{2}{*}{4011.21} & $18.4-26.5$ & 1.00 & 60.5 & $33.3-30.5$ & $3.98-4.40$ & $1.62-1.73$ & & $\mathrm{~B}+$ & $\mathrm{c}$ \\
\hline & & & 22.0 & 1.00 & 35.99 & 32.1 & 4.18 & 0.99 & & $\mathrm{C}$ & $\mathrm{d}$ \\
\hline & ${ }^{2} \mathrm{D}_{5 / 2}^{\mathrm{o}}-{ }^{4} \mathrm{P}_{3 / 2}$ & 4255.60 & $18.4-26.5$ & 1.00 & 27.6 & $41.2-37.3$ & $4.80-5.35$ & $0.60-0.65$ & & A & $\mathrm{c}$ \\
\hline & ${ }^{4} \mathrm{~S}_{3 / 2}^{\mathrm{o}}-{ }^{4} \mathrm{P}_{5 / 2}$ & 4865.91 & $18.4-26.5$ & 1.00 & 68.0 & $50.7-46.0$ & $6.09-6.75$ & $1.20-1.29$ & & $\mathrm{~B}+$ & $\mathrm{c}$ \\
\hline & ${ }^{4} S_{3 / 2}^{o}-{ }^{2} P_{3 / 2}$ & 4535.49 & $18.4-26.5$ & 1.00 & 41.2 & $41.0-39.0$ & $5.01-5.56$ & $0.90-0.92$ & & $\mathrm{~A}$ & $\mathrm{c}$ \\
\hline & ${ }^{2} \mathrm{P}_{1 / 2}^{\mathrm{o}}-{ }^{2} \mathrm{D}_{3 / 2}$ & 2764.65 & 22.0 & 1.00 & 13.79 & 15.5 & 2.00 & 0.79 & & B & $\mathrm{d}$ \\
\hline \multirow[t]{6}{*}{$\left({ }^{1} \mathrm{D}\right) 4 \mathrm{p}-\left({ }^{1} \mathrm{D}\right) 5 \mathrm{~s}$} & \multirow[t]{2}{*}{${ }^{2} \mathrm{~F}_{7 / 2}^{\mathrm{o}}-{ }^{2} \mathrm{D}_{5 / 2}$} & \multirow[t]{2}{*}{3946.10} & $18.4-26.5$ & 1.00 & 33.2 & $34.0-30.9$ & $4.05-4.48$ & $0.87-0.94$ & & $\mathrm{~B}+$ & $\mathrm{c}$ \\
\hline & & & 22.0 & 1.00 & 30.18 & 32.7 & 4.26 & 0.82 & & $\mathrm{~A}$ & $\mathrm{~d}$ \\
\hline & \multirow[t]{3}{*}{${ }^{2} \mathrm{~F}_{5 / 2}^{\mathrm{o}}-{ }^{2} \mathrm{D}_{3 / 2}$} & \multirow[t]{3}{*}{3925.72} & 26.0 & 1.76 & 49.8 & 53.4 & 7.52 & 0.82 & & $\mathrm{~B}+$ & $\mathrm{a}$ \\
\hline & & & $18.4-26.5$ & 1.00 & 31.5 & $33.4-30.2$ & $3.95-4.39$ & $0.84-0.91$ & & A & $\mathrm{c}$ \\
\hline & & & 22.0 & 1.00 & 33.46 & 32.1 & 4.17 & 0.92 & & A & $\mathrm{d}$ \\
\hline & ${ }^{2} \mathrm{~F}_{5 / 2}^{\mathrm{o}}-{ }^{2} \mathrm{D}_{5 / 2}$ & 3926.05 & 22.0 & 1.00 & 33.39 & 32.2 & 4.20 & 0.92 & & A & $\mathrm{d}$ \\
\hline \multirow[t]{3}{*}{$\left({ }^{1} \mathrm{~S}\right) 4 \mathrm{~s}-\left({ }^{3} \mathrm{P}\right) 5 \mathrm{p}$} & ${ }^{2} \mathrm{~S}_{1 / 2}-{ }^{2} \mathrm{D}_{3 / 2}^{\mathrm{o}}$ & 4217.43 & $18.4-26.5$ & 1.00 & 3.6 & $(-15.5)-(-13.8)$ & - & $(-0.23)-(-0.26)$ & & $\mathrm{C}$ & $\mathrm{c}$ \\
\hline & ${ }^{2} \mathrm{~S}_{1 / 2}-{ }^{4} \mathrm{D}_{3 / 2}^{\mathrm{o}}$ & 4385.06 & $18.4-26.5$ & 1.00 & 12.9 & $(-22.3)-(-19.9)$ & - & $(-0.58)-(-0.65)$ & & $\mathrm{C}$ & $\mathrm{c}$ \\
\hline & ${ }^{2} S_{1 / 2}-{ }^{4} S_{3 / 2}^{o}$ & 4189.65 & $18.4-26.5$ & 1.00 & 22.4 & $(-20.1)-(-17.8)$ & - & $(-1.11)-(-1.26)$ & & $\mathrm{B}+$ & $\mathrm{c}$ \\
\hline
\end{tabular}

Table 2. Our calculated wavelengths and oscillator strengths compared with the values taken from NIST database.

\begin{tabular}{|c|c|c|c|c|c|}
\hline Lower level & Upper level & $\begin{array}{c}\lambda(\AA) \\
\text { This work }\end{array}$ & $\begin{array}{l}\lambda(\AA) \\
\text { NIST }\end{array}$ & $\begin{array}{c}f_{i j} \\
\text { This work }\end{array}$ & $\begin{array}{c}f_{i j} \\
\text { NIST }\end{array}$ \\
\hline$\left({ }^{3} P\right) 3 d^{4} D_{3 / 2}$ & $\left({ }^{3} \mathrm{P}\right) 4 \mathrm{p}{ }^{4} \mathrm{P}_{1 / 2}^{\mathrm{o}}$ & 4465.70 & 4332.03 & 0.0320 & 0.027 \\
\hline$\left({ }^{3} \mathrm{P}\right) 3 \mathrm{~d}^{4} \mathrm{D}_{3 / 2}$ & $\left({ }^{3} \mathrm{P}\right) 4 \mathrm{p}^{4} \mathrm{P}_{3 / 2}^{\mathrm{o}}$ & 4524.62 & 4400.01 & 0.0630 & 0.0465 \\
\hline$\left({ }^{3} P\right) 3 d^{4} D_{3 / 2}$ & $\left({ }^{3} \mathrm{P}\right) 4 \mathrm{p}^{4} \mathrm{P}_{5 / 2}^{\mathrm{o}}$ & 4590.42 & 4460.56 & 0.00991 & 0.0067 \\
\hline$\left({ }^{3} P\right) 3 d^{4} D_{7 / 2}$ & $\left({ }^{3} \mathrm{P}\right) 4 \mathrm{p}^{4} \mathrm{P}_{5 / 2}^{\mathrm{o}}$ & 4531.27 & 4400.99 & 0.0890 & 0.066 \\
\hline$\left({ }^{3} P\right) 3 d^{4} D_{5 / 2}$ & $\left({ }^{3} \mathrm{P}\right) 4 \mathrm{p}^{4} \mathrm{P}_{5 / 2}^{\mathrm{o}}$ & 4561.30 & 4431.00 & 0.0490 & 0.0321 \\
\hline$\left({ }^{3} P\right) 3 d^{4} D_{5 / 2}$ & $\left({ }^{3} \mathrm{P}\right) 4 \mathrm{p}^{4} \mathrm{P}_{3 / 2}^{\mathrm{o}}$ & 4496.31 & 4371.32 & 0.0510 & 0.0422 \\
\hline$\left({ }^{3} \mathrm{P}\right) 3 \mathrm{~d}^{4} \mathrm{D}_{5 / 2}$ & $\left({ }^{3} \mathrm{P}\right) 4 \mathrm{p}^{4} \mathrm{D}_{5 / 2}^{\mathrm{o}}$ & 4112.77 & 3986.35 & 0.0151 & 0.011 \\
\hline$\left({ }^{3} \mathrm{P}\right) 3 \mathrm{~d}^{4} \mathrm{D}_{5 / 2}$ & $\left({ }^{3} \mathrm{P}\right) 4 \mathrm{p}^{4} \mathrm{D}_{3 / 2}^{\mathrm{o}}$ & 4037.84 & 3891.97 & 0.0132 & 0.0095 \\
\hline$\left({ }^{3} P\right) 3 d^{4} D_{7 / 2}$ & $\left({ }^{3} \mathrm{P}\right) 4 \mathrm{p}^{4} \mathrm{D}_{7 / 2}^{\mathrm{o}}$ & 4168.04 & 4013.85 & 0.0324 & 0.0254 \\
\hline$\left({ }^{3} \mathrm{P}\right) 3 \mathrm{~d}^{4} \mathrm{D}_{3 / 2}$ & $\left({ }^{3} \mathrm{P}\right) 4 \mathrm{p}{ }^{4} \mathrm{D}_{3 / 2}^{\mathrm{o}}$ & 4060.66 & 3914.77 & 0.0100 & 0.0085 \\
\hline$\left({ }^{3} P\right) 3 d^{4} D_{3 / 2}$ & $\left({ }^{3} \mathrm{P}\right) 4 \mathrm{p}{ }^{4} \mathrm{D}_{1 / 2}^{\mathrm{o}}$ & 4020.95 & 3875.26 & 0.0115 & 0.0092 \\
\hline$\left({ }^{3} \mathrm{P}\right) 3 \mathrm{~d}^{4} \mathrm{~F}_{9 / 2}$ & $\left({ }^{3} \mathrm{P}\right) 4 \mathrm{p}^{4} \mathrm{D}_{7 / 2}^{\mathrm{o}}$ & 6810.11 & 6643.70 & 0.110 & 0.0779 \\
\hline$\left({ }^{3} \mathrm{P}\right) 4 \mathrm{~s}{ }^{4} \mathrm{P}_{5 / 2}$ & $\left({ }^{3} \mathrm{P}\right) 4 \mathrm{p}^{4} \mathrm{P}_{5 / 2}^{\mathrm{o}}$ & 4838.00 & 4806.02 & 0.330 & 0.27 \\
\hline$\left({ }^{3} \mathrm{P}\right) 4 \mathrm{~s}{ }^{4} \mathrm{P}_{3 / 2}$ & $\left({ }^{3} \mathrm{P}\right) 4 \mathrm{p}^{4} \mathrm{P}_{5 / 2}^{\mathrm{o}}$ & 5029.92 & 5009.33 & 0.103 & 0.0853 \\
\hline$\left({ }^{3} \mathrm{P}\right) 4 \mathrm{~s}^{4} \mathrm{P}_{5 / 2}$ & $\left({ }^{3} \mathrm{P}\right) 4 \mathrm{p}{ }^{4} \mathrm{D}_{7 / 2}^{\mathrm{o}}$ & 4426.85 & 4348.06 & 0.531 & 0.4428 \\
\hline$\left({ }^{3} \mathrm{P}\right) 4 \mathrm{p}{ }^{4} \mathrm{P}_{5 / 2}^{\mathrm{o}}$ & $\left({ }^{3} \mathrm{P}\right) 4 \mathrm{~d}^{4} \mathrm{D}_{7 / 2}$ & 3442.59 & 3491.53 & 0.573 & 0.563 \\
\hline$\left({ }^{3} \mathrm{P}\right) 4 \mathrm{p}^{4} \mathrm{P}_{5 / 2}^{\mathrm{o}}$ & $\left({ }^{3} \mathrm{P}\right) 4 \mathrm{~d}^{4} \mathrm{D}_{5 / 2}$ & 3428.10 & 3467.75 & 0.220 & 0.227 \\
\hline$\left({ }^{3} \mathrm{P}\right) 4 \mathrm{p}^{4} \mathrm{D}_{7 / 2}^{\mathrm{o}}$ & $\left({ }^{3} \mathrm{P}\right) 4 \mathrm{~d}^{4} \mathrm{D}_{7 / 2}$ & 3686.67 & 3780.84 & 0.184 & 0.17 \\
\hline ( $\left.{ }^{3} \mathrm{P}\right) 4 \mathrm{p}^{4} \mathrm{D}_{7 / 2}^{\mathrm{o}}$ & $\left({ }^{3} \mathrm{P}\right) 4 \mathrm{~d}^{4} \mathrm{D}_{5 / 2}$ & 3670.07 & 3763.51 & 0.0327 & 0.0284 \\
\hline$\left({ }^{3} \mathrm{P}\right) 4 \mathrm{p}^{4} \mathrm{D}_{7 / 2}^{\mathrm{o}}$ & $\left({ }^{3} \mathrm{P}\right) 4 \mathrm{~d}^{4} \mathrm{~F}_{9 / 2}$ & 3519.71 & 3588.44 & 0.737 & 0.732 \\
\hline$\left({ }^{3} \mathrm{P}\right) 4 \mathrm{p}^{4} \mathrm{D}_{7 / 2}^{\mathrm{o}}$ & $\left({ }^{3} \mathrm{P}\right) 4 \mathrm{~d}^{4} \mathrm{~F}_{7 / 2}$ & 3455.16 & 3521.26 & 0.041 & 0.0422 \\
\hline$\left({ }^{3} \mathrm{P}\right) 4 \mathrm{p}^{4} \mathrm{P}_{5 / 2}^{\mathrm{o}}$ & $\left({ }^{3} \mathrm{P}\right) 5 \mathrm{~s}{ }^{4} \mathrm{P}_{5 / 2}$ & 3736.40 & 3765.27 & 0.17 & 0.21 \\
\hline$\left({ }^{3} \mathrm{P}\right) 4 \mathrm{p}^{4} \mathrm{D}_{7 / 2}^{\mathrm{o}}$ & $\left({ }^{3} \mathrm{P}\right) 5 \mathrm{~s}{ }^{4} \mathrm{P}_{5 / 2}$ & 4025.68 & 4103.91 & 0.189 & 0.23 \\
\hline
\end{tabular}


Table 3. Electron, proton, singly charged helium, and singly charged argon-impact Stark shifts for Ar II lines calculated using semiclassical perturbation approach (Sahal-Bréchot 1969a,b) and a set of atomic data is calculated using Cowan code (Cowan 1981), for a perturber density of $10^{17} \mathrm{~cm}^{-3}$ and temperatures of 5000-60 $000 \mathrm{~K}$. Wavelength of the transitions (in Å) and parameter $C$ (Dimitrijević \& Sahal-Bréchot 1984) are also given. This parameter when divided with the corresponding Stark width gives an estimate for the maximal perturber density for which the line may be treated as isolated. $d_{\mathrm{e}}$ : electron-impact Stark shift, $d_{\mathrm{H}^{+}}$: proton-impact Stark shift, $d_{\mathrm{He}^{+}}$: singly charged helium-impact Stark shift, $d_{\mathrm{Ar}^{+}}$: singly charged argon-impact Stark shift. Cells preceded by an asterisk mean that the impact approximation reaches its limit of validity $(0.1<N V \leq 0.5)$. Empty cells mean that the impact approximation is not valid. This table is available in its entirety for $161 \mathrm{Ar}$ II spectral lines in machine-readable form in the online journal as additional data. A portion is shown here to illustrate its form and content.

\begin{tabular}{|c|c|c|c|c|c|}
\hline Transition & Temperature & $d_{\mathrm{e}}(\AA)$ & $d_{\mathrm{H}^{+}}(\AA)$ & $d_{\mathrm{He}^{+}}(\AA)$ & $d_{\mathrm{Ar}^{+}}(\AA)$ \\
\hline$\left({ }^{3} P\right) 4 p^{4} D_{5 / 2}^{o}-\left({ }^{3} P\right) 4 d^{4} D_{3 / 2}$ & 5000 & 0.393 & $0.237 \mathrm{E}-01$ & $* 0.196 \mathrm{E}-01$ & \\
\hline $3799.38 \AA^{J / 2}$ & 10000 & 0.302 & $0.370 \mathrm{E}-01$ & $* 0.298 \mathrm{E}-01$ & $* 0.222 \mathrm{E}-01$ \\
\hline \multirow[t]{4}{*}{$C=0.61 \mathrm{E}+20$} & 20000 & 0.235 & $0.489 \mathrm{E}-01$ & $0.402 \mathrm{E}-01$ & $* 0.306 \mathrm{E}-01$ \\
\hline & 30000 & 0.210 & $0.551 \mathrm{E}-01$ & $0.450 \mathrm{E}-01$ & $* 0.341 \mathrm{E}-01$ \\
\hline & 40000 & 0.190 & $0.602 \mathrm{E}-01$ & $0.496 \mathrm{E}-01$ & $* 0.378 \mathrm{E}-01$ \\
\hline & 60000 & 0.167 & $0.671 \mathrm{E}-01$ & $0.535 \mathrm{E}-01$ & $* 0.404 \mathrm{E}-01$ \\
\hline$\left({ }^{3} P\right) 4 p^{4} D_{3 / 2}^{o}-\left({ }^{3} P\right) 4 d^{4} D_{1 / 2}$ & 5000 & 0.383 & $0.235 \mathrm{E}-01$ & $* 0.194 \mathrm{E}-01$ & \\
\hline $3841.52 \mathrm{~A}$ & 10000 & 0.290 & $0.367 \mathrm{E}-01$ & $* 0.296 \mathrm{E}-01$ & $* 0.229 \mathrm{E}-01$ \\
\hline \multirow[t]{4}{*}{$C=0.64 \mathrm{E}+20$} & 20000 & 0.226 & $0.486 \mathrm{E}-01$ & $0.397 \mathrm{E}-01$ & $* 0.316 \mathrm{E}-01$ \\
\hline & 30000 & 0.201 & $0.545 \mathrm{E}-01$ & $0.448 \mathrm{E}-01$ & $* 0.352 \mathrm{E}-01$ \\
\hline & 40000 & 0.182 & $0.598 \mathrm{E}-01$ & $0.488 \mathrm{E}-01$ & $* 0.390 \mathrm{E}-01$ \\
\hline & 60000 & 0.159 & $0.658 \mathrm{E}-01$ & $0.528 \mathrm{E}-01$ & $* 0.418 \mathrm{E}-01$ \\
\hline
\end{tabular}

$10^{17} \mathrm{~cm}^{-3}$ and for a set of temperatures from 5000 to $60000 \mathrm{~K}$. In order to take into account the most important perturbers in stellar atmospheres and laboratory plasmas as well as, for example, for argon lasers, Stark shifts are given for electron, proton, singly ionized helium and singly ionized argon-impact broadening. Since our results are obtained using calculated energy levels, a correction to the shifts due to the difference between calculated and observed wavelengths is introduced using equation (8) of Hamdi et al. (2013). All wavelengths given in Table 3 are taken from NIST.

In Table 3, we also specify a parameter $C$ (Dimitrijević \& Sahal-Bréchot 1984), which gives an estimate for the maximal perturber density for which the line may be treated as isolated, when it is divided by the corresponding full width at halfmaximum. For each value given in Table 3, the collision volume $V$ multiplied by the perturber density $N$ is much less than 1 and the impact approximation is valid (Sahal-Bréchot 1969a,b). For $N V>0.5$, the impact approximation breaks down and thus the values are not given. For $0.1<N V \leq 0.5$, the impact approximation reaches his limit of validity and values are preceded by an asterisk.

All the obtained Stark shifts presented in Table 3 will be implemented in STARK-B database (http://stark-b.obspm.fr/; Sahal-Bréchot et al. 2015). STARK-B is a node of the Virtual Atomic and Molecular Data Center (Dubernet et al. 2016; http://www.vamdc.org/).

\section{CONCLUSIONS}

We have performed semiclassical perturbation calculations of Stark shifts for singly charged argon spectral lines. Energy levels and oscillator strengths are calculated using Hartree-Fock method with relativistic correction (HFR) (Cowan 1981). Our Stark shifts are presented as a function of temperature for 161 spectral lines for collisions with electrons, protons, singly ionized helium, and singly ionized argon. Our results are compared with experimental values for 100 spectral lines taking into account collisions with ions. Our results are generally in good agreement with the experimental results and with the theoretical values of Griem (1974) also. Our Stark shifts may be of interest for diagnostics of plasma laboratories, spectral calibration in laboratories and modelling of A, B, and subdwarf stars. New experimental results and accurate quantum mechanical results will be of interest for a better investigation of Stark shifts in Ar II spectrum.

\section{ACKNOWLEDGEMENTS}

This work is part of the project 176002 'Influence of collisional processes on astrophysical plasma line shapes' supported by the Ministry of Education, Science and Technological Development of Serbia. This work has been also supported by the Paris Observatory, the CNRS and the PNPS (Programme National de Physique Stellaire, INSU-CNRS), France. This work has also been supported by the LABEX Plas@par project, and received financial state aid managed by the Agence Nationale de la Recherche, as part of the programme 'Investissements d'avenir' under the reference ANR11-IDEX-0004-02.

\section{REFERENCES}

Aparicio J. A., Gigosos M. A., González V. R., Pérez C., de la Rosa M. I., Mar S., 1998, J. Phys. B, 31, 1029

Blanchette J.-P., Chayer P., Wesemael F., Fontaine G., Fontaine M., Dupuis J., Kruk J. W., Green E. M., 2008, ApJ, 678, 1329

Cowan R. D., 1981, The Theory of Atomic Structure and Spectra. Univ. California Press, Berkeley, USA

Dimitrijević M. S., Sahal-Bréchot S., 1984, J. Quant. Spectrosc. Radiat. Transfer, 31, 301

Dimitrijević M. S., Sahal-Bréchot S., 1996, Phys. Scr., 54, 50

Dimitrijević M. S., Sahal-Bréchot S., 2014, Atoms, 2, 357

Dimitrijević M. S., Sahal-Bréchot S., Bommier V., 1991, A\&AS, 89, 581

Dimitrijević M. S., Simić Z., Kovačević A., Valjarević A., Sahal-Bréchot S., 2015, MNRAS, 454, 1736

Djeniže S., Malešević M., Srécković A., Milosavljević M., Purić J., 1989, J. Quant. Spectrosc. Radiat. Transf., 42, 429 
Djurović S., Belmonte M. T., Peláez R. J., Aparicio J. A., Mar S., 2013, MNRAS, 433, 1082

Dubernet M. L. et al., 2016, J. Phys. B, 49, 074003

Dzierzega K., Musiol K., 1994, J. Quant. Spectrosc. Radiat. Transf., 52, 747

Fleurier C., Sahal-Bréchot S., Chapelle J., 1977, J. Quant. Spectrosc. Radiat. Transfer, 17, 595

Fossati L., Ryabchikova T., Bagnulo S., Alecian E., Grunhut J., Kochukhov O., Wade G., 2009, A\&A, 503, 945

Griem H. R., 1974, Spectral Line Broadening by Plasmas. Academic Press, New York

Hamdi R., Ben Nessib N., Milovanović N., Popović L. Č., Dimitrijević M. S., Sahal-Bréchot S., 2008, MNRAS, 387, 871

Hamdi R., Ben Nessib N., Dimitrijević M. S., Sahal-Bréchot S., 2011, Baltic Astron., 20, 552

Hamdi R., Ben Nessib N., Dimitrijević M. S., Sahal-Bréchot S., 2013, MNRAS, 431, 1039

Hamdi R., Ben Nessib N., Sahal-Bréchot S., Dimitrijević M. S., 2014, Adv. Space Res., 54, 1223

Hamdi R., Ben Nessib N., Sahal-Bréchot S., Dimitrijević M. S., 2017, Atoms, 5, 26

Hamdi R., Ben Nessib N., Sahal-Bréchot S., Dimitrijević M. S., 2018, MNRAS, 475, 800

Heber U., Edelmann H., 2004, Ap\&SS, 291, 341

Khokhlova V. L., 1994, Astron. Lett., 20, 89

Konjević N., Lesage A., Fuhr J. R., Wiese W. L., 2002, J. Phys. Chem. Ref. Data, 31, 819

Kramida A., Ralchenko Yu., Reader J., NIST ASD Team, 2018, NIST Atomic Spectra Database (ver. 5.5.6). National Institute of Standards and Technology, Gaithersburg, MD. Available at: https://physics.nist.g ov/asd

Lanz T., Cunha K., Holtzman J., Hubeny I., 2008, ApJ, 678, 1342

Lesage A., 2009, New Astron. Rev., 52, 471

Majlinger Z., Simić Z., Dimitrijević M. S., 2017, MNRAS, 470, 1911

Sahal-Bréchot S., 1969a, A\&A, 1, 91
Sahal-Bréchot S., 1969b, A\&A, 2, 322

Sahal-Bréchot S., 1974, A\&A, 35, 319

Sahal-Bréchot S., 1991, A\&A, 245, 322

Sahal-Bréchot S., Dimitrijević M. S., Ben Nessib N., 2014, Atoms, 2, 225

Sahal-Bréchot S., Dimitrijević M. S., Moreau N., Ben Nessib N., 2015, Phys. Scr., 90, 054008

Simić Z., Dimitrijević M. S., Milovanović N., Sahal-Bréchot S., 2005, A\&A, 441,391

Simić Z., Dimitrijević M. S., Sahal-Bréchot S., 2013, MNRAS, 432, 2247

Vennes S., Kawka A., Németh P., 2011, MNRAS, 413, 2545

Werner K., Rauch T., Kruk J. W., 2016, A\&A, 593, A104

Werner K., Rauch T., Kruk J. W., 2017, A\&A, 601, A8

Werner K., Rauch T., Kruk J. W., 2018, A\&A, 616, A73

Wiese W. L., Kelleher D. E., 1971, ApJ, 166, L59

\section{SUPPORTING INFORMATION}

Supplementary data are available at MNRAS online.

Table 3. Electron, proton, singly charged helium, and singly charged argon-impact Stark shifts for Ar II lines calculated using semiclassical perturbation approach (Sahal-Bréchot 1969a,b) and a set of atomic data is calculated using Cowan code (Cowan 1981), for a perturber density of $10^{17} \mathrm{~cm}^{-3}$ and temperatures of 5000 $60000 \mathrm{~K}$.

Please note: Oxford University Press is not responsible for the content or functionality of any supporting materials supplied by the authors. Any queries (other than missing material) should be directed to the corresponding author for the article.

This paper has been typeset from a $\mathrm{T}_{\mathrm{E}} \mathrm{X} / \mathrm{L} \mathrm{T} \mathrm{E} \mathrm{X}$ file prepared by the author. 